\title{
Bortezomib in the management of multiple myeloma
}

This article was published in the following Dove Press journal:

Cancer Management and Research

7 September 2009

Number of times this article has been viewed

\author{
Jacob P Laubach \\ Constantine S Mitsiades \\ Teru Hideshima \\ Robert Schlossman \\ Dharminder Chauhan \\ Nikhil Munshi \\ Irene Ghobrial \\ Nicole Carreau \\ Kenneth C Anderson \\ Paul G Richardson \\ Department of Medical Oncology, \\ Dana Farber Cancer Institute, Boston, \\ Massachusetts, USA
}

Correspondence: Jacob P Laubach Dana Farber Cancer Institute, Boston, MA 02II5, USA

$\mathrm{Tel}+\mid 6176325103$

Fax +I 6175828608

Email jacobp_laubach@dfci.harvard.edu
Abstract: Multiple myeloma (MM) is a B-cell malignancy characterized by clonal expansion of plasma cells within the bone marrow, the presence of a serum and/or urine monoclonal protein, lytic bone lesions, and anemia. On a cellular level, the disease is characterized by complex interactions between tumor cells and the surrounding bone marrow microenvironment. Understanding of the relationship between malignant plasma cells and the microenvironment has sparked ongoing efforts to develop targeted therapeutic agents for treatment of this disease. The successful development of the first-in-class small-molecule proteasome inhibitor bortezomib occurred as a result of these efforts. This review focuses on the rationale for bortezomib therapy in the treatment of patients with newly diagnosed and relapsed MM, important treatment-related side effects, and future directions for use of bortezomib and other, emerging proteasome inhibitors.

Keywords: multiple myeloma, bortezomib, stem cell transplantation, peripheral neuropathy

\section{Introduction}

Multiple myeloma (MM) is a hematologic malignancy of B-cell origin that constitutes approximately $1 \%$ of all malignant tumors and $10 \%$ to $15 \%$ of hematopoietic neoplasms. ${ }^{1}$ In 2008, there were an estimated 19,920 new cases of MM in the United States and 10,690 deaths attributable to the disease. ${ }^{2}$ It primarily occurs in older individuals, with an average age at diagnosis of $65,{ }^{3}$ and is associated with a number of important clinical manifestations including osteolytic bone lesions, renal failure, anemia, recurrent infections, neuropathy, and hypercalcemia. For over 40 years, corticosteroids and conventional chemotherapy provided the basis for MM therapy with such regimens as high-dose dexamethasone; ${ }^{4,5}$ melphalan and prednisone; ${ }^{6}$ and vincristine, doxorubicin, and pulsed high-dose dexamethasone (VAD). ${ }^{7-11}$ High-dose therapy and autologous stem cell transplantation (ASCT) in MM was pioneered during the $1980 \mathrm{~s}^{12,13}$ and has a well established role in the treatment of appropriately selected individuals with MM. Over the past decade, though, the therapy for MM has changed significantly with the introduction of the immunomodulatory drugs (IMiDs) thalidomide and lenalidomide as well as the proteasome inhibitor bortezomib. These agents target specific pathways within MM cells and the bone marrow microenvironment that have been identified and characterized through careful preclinical investigation. This review focuses specifically on the development and current applications of the first-in-class proteasome inhibitor bortezomib in the treatment of MM. 


\section{Mechanism of action}

The ubiquitin-proteasome pathway plays an important role in intracellular protein homeostasis by regulating protein degradation. As such, it affects critical cellular processes such as cell cycle regulation, antigen processing, and apoptosis. Protein degradation occurs through a 2-step process in which proteins destined for removal first undergo ATP-dependent ubiquitination followed by a second step of proteolysis within the $26 \mathrm{~S}$ proteasome, which consists of a proteolytic core, the $20 \mathrm{~S}$ proteasome, surrounded by two 19S regulatory complexes. ${ }^{14}$ The $20 \mathrm{~S}$ proteasome possesses chymotrypsin-like, trypsin-like, and caspase-like catalytic activity. Proteasome inhibitors are classified as either reversible or irreversible and on the basis of their inhibition of chymotrypsin-like, trypsin-like, or caspase-like catalytic activity.

Bortezomib is a boronic acid dipeptide small molecule that reversibly inhibits the chymotrypsin-like activity of the 20S proteasome. The onset of bortezomib's anti-MM activity is rapid, with apoptosis of MM cells occurring within several hours after exposure. ${ }^{15} \mathrm{NF}-\mathrm{\kappa B}$ is an important target of bortezomib within MM cells. Inhibition of NF- $\kappa B$ decreases adherence of MM cells to the bone marrow stromal cells, thus inhibiting paracrine-mediated growth of MM cells and enhancing susceptibility to therapeutic agents. ${ }^{16}$ While inhibition of NF- $\mathrm{KB}$ provided the initial rationale for use of this agent in MM, it is now understood that bortezomib targets a number of other molecules and pathways within MM cells. For example, bortezomib-induced apoptosis of MM cells is associated with activation of caspase-8/9 and caspase-3. ${ }^{15}$ Bortezomib also cleaves DNA repair enzymes, increasing the susceptibility of MM cells to classes of DNA-damaging agents such as alkylating agents and anthracyclines. ${ }^{17,18}$ In addition, the agent induces pro-apoptotic elements of the unfolded protein response such as PERK, ATF4, and CHOP/GADD153. ${ }^{19,20}$ Finally, IL-6- induced activation of ERK, STAT3, and AKT is inhibited by bortezomib via its ability to downregulate gp $130 .{ }^{21}$

In addition to these anti-tumor effects, bortezomib has important effects on the development and progression of MM-associated bone disease. Bone abnormalities such as osteoporosis, compression fractures, and lytic lesions are characteristic of MM and are present in approximately $80 \%$ of patients at the time of diagnosis. ${ }^{22} \mathrm{MM}$ cells secrete osteoclast activating factors such as RANKL, IL-3, macrophage inflammatory protein, and IL-6, while levels of the RANKL decoy receptor osteoprotegrin, which regulates osteoclast activity, are decreased in patients with the disease. ${ }^{23}$ In addition, increased levels of osteoblast inhibitors dickhofp-1 (DKK1), IL-7, and IL-3 in MM diminish bone anabolism. ${ }^{24}$ These events disrupt the balance of osteoblast and osteoclast function in favor of bone catabolism and contribute to the pathogenesis of MM-associated bone disease. Bortezomib antagonizes these processes in several ways. It decreases levels of RANKL and DKK $1^{25}$ and increases levels of alkaline phosphatase and osteocalcin, two markers of bone formation. ${ }^{26}$ In addition, bortezomib appears to inhibit osteoclast differentiation ${ }^{27}$ and augment osteoblast proliferation by inducing the differentiation of mesenchymal stem cells into osteoblasts. ${ }^{28}$ The beneficial effects of bortezomib on bone disease in MM have also been demonstrated in vivo through studies utilizing murine models..$^{29,30}$

\section{Bortezomib-based treatment strategies \\ Relapsed MM}

The significant anti-MM activity of bortezomib observed in preclinical studies provided the impetus for subsequent clinical development of the drug. Phase I and II studies involving patients with relapsed MM demonstrated a manageable toxicity profile and confirmed the activity of bortezomib in this setting. ${ }^{31-33}$ These efforts culminated in the international, multicenter phase III Assessment of Proteasome Inhibtion for Extending Remissions (APEX) trial, in which 669 patients with relapsed MM, more than $50 \%$ of whom had undergone two or more prior lines of therapy, were randomized to receive either bortezomib $1.3 \mathrm{mg} / \mathrm{m}^{2}$ on days $1,4,8$, and 11 of each 21 -day cycle for eight 3-week cycles, followed by treatment on days 1 , 8,15 , and 22 for four 5-week cycles; or dexamethasone $40 \mathrm{mg}$ on days $1-4,9-12,17-20$ for four 5 -week cycles, followed by treatment on days $1-4$ for five 4 -week cycles. ${ }^{34}$ Bortezomib was superior to high-dose dexamethasone for overall response (OR) rate (38\% vs $18 \%$ ), complete response (CR) rate ( $6 \%$ vs $1 \%)$, median time to progression (TTP) (6.22 vs 3.49 months), and 1-year overall survival (OS) $(80 \%$ vs $66 \%)$. Grade $3 / 4$ treatment-related toxicities included thrombocytopenia (26\%), neutropenia (14\%), anemia (10\%), peripheral neuropathy $(7 \%)$, and diarrhea (7\%). With extended follow up of APEX participants, the OR and $\mathrm{CR}$ rates among bortezomib-treated patients improved to $43 \%$ and $9 \%$, respectively, ${ }^{35}$ while the median OS was 29.8 months in the bortezomib arm compared to 23.7 months in the dexamethasone arm.

Demonstration in preclinical studies of synergy between bortezomib and other classes of agents such as corticosteroids, 
alkylating agents, and anthracyclines prompted evaluation of bortezomib-based combinations. ${ }^{15,17,36}$ In the phase II Study of Uncontrolled Myeloma Management with proteasome Inhibition Therapy (SUMMIT) and Clinical Response and Efficacy Study of bortezomib in the Treatment of refractory myeloma (CREST) trials, patients with progressive disease after two cycles or stable disease after four cycles could receive oral dexamethasone $20 \mathrm{mg}$ on the day of and day after bortezomib. In the SUMMIT trial, 13 (18\%) of 78 patients with stable or progressive disease after several cycles of bortezomib monotherapy achieved a minimal or partial response with the combination. ${ }^{32}$ Among CREST study participants, the OR rate among those who received the combination was $50 \%{ }^{33}$

As predicted by preclinical models, combinations including bortezomib and anthracyclines have also been effective in relapsed and refractory MM. In a randomized, phase III trial involving 646 individuals with relapsed and refractory disease, $66 \%$ of whom had received two or more prior lines of therapy, treatment with bortezomib plus liposomal doxorubicin was superior to bortezomib alone in terms of median TTP (9.3 vs 6.5 months) and 15 month OS (76\% vs 65\%). ${ }^{37}$ Although grade $3 / 4$ toxicities such as anorexia, vomiting, thrombocytopenia, neutropenia, and hand-foot syndrome occurred more frequently with the doublet, cardiac toxicity was only minimally increased with the combination and rates of peripheral neuropathy (PN) were nearly equivalent. In a phase II study involving 64 heavily pretreated patients with relapsed and refractory MM, bortezomib, doxorubicin, and dexamethasone (PAD) produced a partial response (PR) or better in $67 \%$ and a very good partial response (VGPR) or better in $25 \% .{ }^{38}$ Frequent grade 3/4 toxicities included thrombocytopenia, neutropenia, infection, and peripheral neuropathy, and two patients experienced grade $3 / 4$ congestive heart failure.

In addition to its sensitizing effect on corticosteroids and conventional classes of chemotherapeutic agents such as anthracyclines, bortezomib exhibits significant activity in combination with other novel agents in the treatment of relapsed and refractory MM. In a phase I/II study, bortezomib and thalidomide were administered to 85 patients with relapsed and refractory $\mathrm{MM} \cdot{ }^{39}$ The dose range of bortezomib was $1.0-1.3 \mathrm{mg} / \mathrm{m}^{2}$ on days $1,4,8$, and 11 , while thalidomide was given starting with cycle two at doses of 50-200 mg/day. Dexamethasone $20 \mathrm{mg}$ on the day of and day after bortezomib was added during cycle four for patients with less than a PR. A minor response (MR) or better occurred in $79 \%$ of study participants, while $63 \%$ achieved a PR or better. The most common grade 3/4 toxicities included thrombocytopenia and neutropenia. Although the cumulative incidence of PN with this combination has been approximately $60 \%$, grade 3/4 PN has been infrequent and the neuropathy has, in many instances, been reversible.

Bortezomib plus lenalidomide, and dexamethasone (RVD) is also very active in relapsed and refractory MM. The rationale for this approach is derived from preclinical work demonstrating dual apoptotic signaling, with in vitro modeling suggesting the synergistic tumoricidal activity of bortezomib and lenalidomide. ${ }^{40}$ In a phase II study, 64 patients with relapsed and refractory $\mathrm{MM}$ who had received 1-3 prior lines of therapy received bortezomib $1.0 \mathrm{mg} / \mathrm{m}^{2}$ days $1,4,8$, and 11; lenalidomide $15 \mathrm{mg}$ days $1-14$, and dexamethasone $40 \mathrm{mg}$ (cycles 1-4) or $20 \mathrm{mg}$ (cycles 5-8) on days of, and after, bortezomib for up to eight 21-day cycles. ${ }^{41}$ To date, the rate of MR or better in this study is $86 \%$, with $24 \%$ of study participants achieving a $\mathrm{CR} /$ near $\mathrm{CR}$ (nCR) and 67\% achieving a PR or better. Among patients who respond to therapy, the median duration of response (DOR) has been 21 weeks. Importantly, response rates have been equivalent among patients with standard risk features and those with high-risk disease characterized by advanced ISS stage and cytogenetic abnormalities. Toxicities have included grade 1-2 myelosuppression and two cases of deep vein thrombosis (DVT).

\section{Newly diagnosed MM}

Bortezomib has also proven effective in the treatment of patients with newly diagnosed MM on the basis of numerous clinical trials involving patients who are eligible for ASCT and those who are not. In a large, multicenter phase III trial involving 682 ASCT-ineligible patients with newly diagnosed MM, bortezomib plus melphalan and prednisone (VMP) was compared to MP alone. ${ }^{42}$ All patients received melphalan $9 \mathrm{mg} / \mathrm{m}^{2}$ and prednisone $60 \mathrm{mg} / \mathrm{m}^{2}$ days $1-4$ of each 6-week cycle, while bortezomib was administered in the VMP arm at $1.3 \mathrm{mg} / \mathrm{m}^{2}$ on days $1,4,8,11,22,25,29$, and 32 during cycles $1-4$ and on days 1, 8, 22, and 29 during cycles 5-9. VMP was superior to MP in terms of the study's primary endpoint of TTP (24 vs 16.6 months), as well as secondary endpoints CR rate (30\% vs 4\%) and DOR (19.9 vs 13.1 months). The hazard ratio for survival favored VMP to MP (0.61). Grade 3 toxicities were more common with VMP than MP (53\% vs 44\%), while grade 4 toxicities were equivalent ( $28 \%$ vs $27 \%$ ). $13 \%$ of participants in the VMP arm experienced grade $3 \mathrm{PN}$, while one patient developed grade $4 \mathrm{PN}$. 
A variety of bortezomib-based combinations have been utilized in the upfront treatment of individuals eligible for ASCT. In one of the largest of these trials, 480 patients with newly diagnosed MM were randomized to either VAD or bortezomib plus dexamethasone induction. ${ }^{43}$ This was followed by a second randomization to two cycles of dexamethasone, cyclophosphamide, etoposide, platinum (DCEP), consolidation or not, prior to ASCT. In preliminary analysis, bortezomib plus dexamethasone was superior to VAD induction with respect to rates of VGPR or better ( $46.7 \%$ vs $18.6 \%)$ and $\mathrm{CR} / \mathrm{nCR}(21.3 \%$ vs $8.3 \%)$, even among patients with an advanced ISS score and del(13). Importantly, the benefit of bortezomib-based induction in this trial persisted post-ASCT with respect to both VGPR or better $(40.8 \%$ vs $28.8 \%)$ and $\mathrm{CR} / \mathrm{nCR}(71.8 \%$ vs $51 \%)$. DCEP consolidation did not improve response rates in either treatment group. Although treatment-related PN occurred more frequently with bortezomib plus dexamethasone induction, the overall rate of therapy-related toxicities was equivalent. Stem cell collection was successful in $97 \%$ of patients who received bortezomib plus dexamethasone and $99 \%$ of those who received VAD.

In another large, phase III trial, 480 transplant-eligible patients with newly diagnosed MM were randomized to bortezomib plus thalidomide and dexamethasone (VTD) or thalidomide and dexamethasone (TD) alone. Patients in the TD arm received thalidomide $200 \mathrm{mg}$ daily for days $1-63$ with dexamethasone $40 \mathrm{mg}$ daily on days $1-4$ and 9-12 of each 21-day cycle. Patients in the VTD arm received the same dose/schedule of thalidomide, with bortezomib $1.3 \mathrm{mg} / \mathrm{m}^{2}$ on days $1,4,8$, and 11 of each cycle and dexamethasone $40 \mathrm{mg}$ on the day of, and after, each dose of bortezomib. ${ }^{44}$ Preliminary analysis presented at the 2008 American Society of Hematology (ASH) meeting demonstrated the superiority of VTD in comparison to TD with respect to OR rate $(92 \%$ vs $78.5 \%), \mathrm{CR} / \mathrm{nCR}$ rate ( $33 \%$ vs $12 \%$ ), and VGPR or better $(61 \%$ vs $30 \%)$. While the rate of $\geq$ grade $3 \mathrm{PN}$ was higher in the VTD arm ( $9 \%$ vs $2.5 \%$ ), the overall rate of serious adverse events was similar. A sufficient number of stem cells for up to two ASCT were collected in $91 \%$ of patients in the VTD group and $87 \%$ in the TD arm. Among patients who underwent ASCT, those who received VTD did better in terms of VGPR or better ( $75 \%$ vs $53 \%$ ), CR/nCR ( $54 \%$ vs $29 \%$ ), and CR rate ( $41 \%$ vs $20 \%$ ). After 15 months of follow-up, progression-free survival (PFS) was superior among study participants who received VTD (93\% vs 86\%), while the 20-month overall survival rate was equivalent in the two arms.
In the phase III HOVON-65/GMMG-HD4 study, preliminary results of which were also presented at the 2008 ASH annual meeting, 833 transplant-eligible patients with newly diagnosed MM were randomized to either VAD or PAD induction followed by stem cell mobilization and either single or tandem ASCT. ${ }^{45}$ This was followed by maintenance therapy with either thalidomide $50 \mathrm{mg}$ daily (VAD arm) or bortezomib $1.3 \mathrm{mg} / \mathrm{m}^{2}$ every 2 weeks (PAD arm) for 2 years. In a preliminary analysis, PAD induction proved superior to VAD with respect to rates of OR ( $80 \%$ vs $64 \%$ ), VGPR or better ( $41 \%$ vs $17 \%)$, and CR ( $5 \%$ vs $0 \%$ ). The benefit of PAD over VAD induction persisted post-transplant, with superior OR ( $92 \%$ vs $77 \%$ ) and CR (15\% vs $4 \%$ ) rates. In this initial analysis, bortezomib maintenance further improved the $\mathrm{CR} / \mathrm{nCR}$ rate from $23 \%$ to $35 \%$, suggesting that additional bortezomib-based therapy post-transplant deepens the overall response to therapy.

RVD is also highly effective as initial therapy for both transplant-eligible and transplant ineligible patients with newly diagnosed MM. In a phase I/II study of this combination, 68 patients have received the combination to date, 33 as part of the phase I dose-escalation phase and 35 in the phase II phase utilizing the maximum tolerated dose (MTD) of lenalidomide $25 \mathrm{mg}$ and bortezomib $1.3 \mathrm{mg} / \mathrm{m}^{2} .{ }^{46,47}$ The OR rate is $100 \%$, with $74 \%$ of patients achieving a VGPR or better and $44 \%$ achieving a $\mathrm{CR} / \mathrm{nCR}$. Among patients who received the MTD, the OR rate was $100 \%$. It is notable that rates of response were not affected by such adverse prognostic features as del $(13)$ and $t(4 ; 14)$. Moreover, the regimen was well-tolerated, with low rates of both DVT/ pulmonary embolism (5\%) and grade $\geq 3 \mathrm{PN}(3 \%)$.

\section{Bortezomib-based therapy in unique patient populations}

Coupled with advances in translational science, rapid drug development in MM has substantially expanded treatment options for patients. This is underscored by the most recent guidelines on MM therapy from the National Comprehensive Cancer Network (NCCN), which include multiple treatment regimens for both relapsed and refractory disease and newly diagnosed $\mathrm{MM} .^{48}$ Various factors are considered in decisions on appropriate therapy for an individual patient, including the clinical/biological features of a patient's disease, comorbid conditions, and mode of drug administration. Clinical experience with bortezomib indicates there are certain patients in whom the agent appears to confer unique benefits.

Bortezomib appears to overcome the poor prognosis associated with an elevated $\mathrm{B}_{2}$-microglobulin ${ }^{49}$ and with 
chromosomal abnormalities such as $13 \mathrm{q}$ deletion and $\mathrm{t}(4 ; 14) .{ }^{49-52}$ The agent is thus an important component of therapy for individuals with high-risk MM on the basis of these prognostic factors. Bortezomib has also proven effective in the management of MM patients with renal dysfunction, a common manifestation of the disease noted at the time of diagnosis in approximately $30 \%$ of individuals. ${ }^{22,53}$ Based on the results of a case series involving MM patients with dialysis-requiring renal failure at the time of bortezomib therapy, the agent yields OR and CR rates in this population that are comparable to those seen among individuals without renal failure.$^{54}$ Moreover, four patients in this series became dialysis-independent as a result of bortezomib therapy. As discussed previously, bortezomib therapy is beneficial for individuals with significant disease-related bone disease due to its inhibitory effect on osteoclastogenesis ${ }^{27}$ and stimulatory effect on osteoblast differentiation and proliferation. ${ }^{28}$ Finally, MM patients with associated AL amyloidosis can be considered for bortezomib-based therapy on the basis of preliminary evidence of its efficacy in this setting. In a retrospective analysis of 20 patients with previously treated systemic AL amyloidosis who received bortezomib on a standard treatment schedule, 16 responded, ${ }^{55}$ with amyloidotic organ function improved in 6 of the 16 patients who responded to therapy.

\section{Treatment-related side effects}

Optimization of therapeutic benefit with bortezomib use requires familiarity with, and appropriate management of, treatment-related side effects associated with the agent. Fatigue, diarrhea, PN, thrombocytopenia, and herpes zoster reactivation are among those that are monitored throughout the course of therapy.

\section{Peripheral neuropathy}

Initial symptoms associated with bortezomib-induced PN include pain involving the distal extremities along with sensory dysfunction resulting from small-fiber axonal injury. With progression of $\mathrm{PN}$, proprioceptive loss, distal weakness of the upper and lower extremities, and suppression of deep tendon reflexes may occur. ${ }^{56,57}$ Among 256 patients enrolled in the phase II SUMMIT and CREST trials, 90 (35\%) developed treatment-emergent $\mathrm{PN}$ or exacerbation of pre-existing PN. ${ }^{58}$ The incidence of PN was dose-related, occurring more frequently at the $1.3 \mathrm{mg} / \mathrm{m}^{2}$ dose than $1.0 \mathrm{mg} / \mathrm{m}^{2}$, and peaked at cycle 5 with a cumulative dose of approximately $30 \mathrm{mg} / \mathrm{m}^{2}$. While the rate of bortezomib-associated PN was similar among patients with and without baseline PN, patients with pre-existing PN experienced more severe treatment-related symptoms. One or more courses of therapy was withheld as a result of treatment-associated PN in 19/90 (21\%) patients, while dose reduction or discontinuation were required in $12 \%$ and $5 \%$, respectively. Bortezomib-associated PN is reversible with treatment interruption in the majority of patients. ${ }^{58,59}$ Indeed, among SUMMIT and CREST study participants, symptoms improved to baseline in $71 \%$ of those who experienced $\geq$ grade 3 treatment-associated PN.

Various measures can be employed to prevent bortezomibinduced PN and manage symptoms when PN occurs. Alpha-lipoic acid, an organosulfur enzyme cofactor that possesses antioxidant properties and modulates glucose uptake, has been used for prevention of diabetic peripheral neuropathy and may be beneficial for MM patients who receive bortezomib, although this intervention requires further study in bortezomib-treated MM patients. ${ }^{60}$ Similarly, acetyl-L-carnitine, an ammonium-containing compound derived from the amino acids methionine and lysine, has antioxidant and neurotrophic activity and appears to ameliorate chemotherapy-induced PN. ${ }^{61}$ In addition, the regular application of thick emollients enriched with antioxidants and putative neurotransmitters, such as cocoa butter or topical menthol-containing preparations, ${ }^{62}$ appears to benefit patients receiving bortezomib, perhaps by enhancing small fiber function and recovery. There is solid clinical rationale for these interventions, although prospective studies are needed to validate their efficacy in the context of bortezomib-based MM therapy.

Despite such strategies aimed at limiting the development or progression of bortezomib-induced PN, a significant number of $\mathrm{MM}$ patients require additional intervention. A prospective algorithm for bortezomib dose reductions as was derived from the SUMMIT and CREST trials and applied in the APEX study is recommended for individuals who receive the agent (Table 1). ${ }^{34}$ Patients with pain or severe paresthesias may require symptom-directed pharmacotherapy. Options that have proven effective in this respect include the anticonvulsants gabapentin and pregabalin; serotonin-norepinephrine reuptake inhibitors such as duloxetine; tricyclic antidepressants amitriyptyline and desipramine; and opioids such as oxycodone, morphine sulfate, and hydrocodone. ${ }^{63}$

\section{Thrombocytopenia}

In the SUMMIT and CREST trials, therapy-associated thrombocytopenia occurred in $42 \%$ of study participants, and was the most frequently reported grade 3 adverse event. ${ }^{64}$ 
Table I Dose modification guideline for bortezomib-related neuropathic pain and/or peripheral sensory or motor neuropathy ${ }^{10}$

\begin{tabular}{ll}
\hline Severity of peripheral neuropathy signs and symptoms & Modification of bortezomib dose and regimen \\
\hline Grade I (paresthesias, weakness and/or loss of reflexes) without pain or loss of function & No action \\
$\begin{array}{l}\text { Grade I with pain or Grade } 2 \text { (interfering with function but not with activities of } \\
\text { daily living) }\end{array}$ & Reduce to $1.0 \mathrm{mg} / \mathrm{m}^{2}$ \\
Grade 2 with pain or Grade 3 (interfering with activities of daily living) & Withhold treatment until toxicity resolves, then reinitiate \\
& $\begin{array}{l}\text { at a dose of } 0.7 \mathrm{mg} / \mathrm{m}^{2} \text { once weekly } \\
\text { Grade } 4 \text { (sensory neuropathy that is disabling or motor neuropathy that }\end{array}$ \\
is life-threatening or leads to paralysis) & Discontinue
\end{tabular}

Notes: Grading for this currently recommended dose modification guideline is based on National Cancer Institute Common Terminology Criteria for Adverse Events ( $\mathrm{NCl} \mathrm{CTCAE)} \mathrm{version} \mathrm{3.0.} \mathrm{In} \mathrm{APEX,} \mathrm{the} \mathrm{dose} \mathrm{modification} \mathrm{guideline} \mathrm{used} \mathrm{was} \mathrm{the} \mathrm{same,} \mathrm{but} \mathrm{based} \mathrm{on} \mathrm{NCl} C \mathrm{CTC}$ version 2.0 grading; in addition, patients experiencing grade 3 peripheral neuropathy with pain were to discontinue bortezomib.

Grade 3/4 thrombocytopenia was noted in 30\% of study participants overall but importantly was uncommon (13\%) in individuals with a baseline platelet count $>200 \times 10^{9} / \mathrm{L}$. Indeed, there was an inverse correlation between baseline platelet count and the incidence of grade 3/4 thrombocytopenia. Bleeding episodes associated with bortezomib-induced thrombocytopenia were infrequent, but can occur as a complication of therapy, albeit rarely. In one instance, gastrointestinal bleeding developed in the setting of grade 3 thrombocytopenia. In another, grade 1 epistaxis occurred in the context of grade 4 thrombocytopenia.

Although the mechanism by which bortezomib induces thrombocytopenia is unknown, murine studies suggest that it does involve a direct cytotoxic effect on megakaryocytes or alteration in thrombopoietin (TPO) levels. ${ }^{64}$ During a standard 21-day treatment cycle, the platelet count typically follows a biphasic pattern, with a decline of on average $60 \%$ of the baseline platelet count during the 11-day period of bortezomib administration followed by recovery during the rest period. ${ }^{64}$ The platelet count is thus monitored closely during treatment, with platelet transfusions if indicated and bortezomib dose reduction for high grade thrombocytopenia if it is persistent despite transfusion and if there is a concern regarding hemorrhage. The presence of baseline thrombocytopenia and concomitant use of agents known to cause myelosuppression - such as anthracyclines, alkylating drugs, and lenalidomide - necessitates caution.

\section{Gastrointestinal side effects}

Gastrointestinal (GI) side effects associated with bortezomib were common among participants in the APEX trial, with diarrhea and nausea occurring in $57 \%$, constipation in $42 \%$, vomiting in $35 \%$, anorexia in $23 \%$, and abdominal pain in $16 \% .{ }^{34}$ However, grade $3 / 4$ bortezomib-associated GI toxicities were infrequent in this study. Pre-emptive supportive care measures are an important component of care in the management of MM patients receiving bortezomib. Corticosteroids, $5-\mathrm{HT}_{3}$ receptor antagonists such as ondansetron and phenothiazines are effective antiemetics in this setting. Stool softeners, laxatives, and antidiarrheals are utilized in the management of constipation and/or diarrhea. Proton-pump inhibitors and/or H2-receptor blockers are employed in patients who, due to prolonged steroid use, are prone to gastritis or gastric/duodenal ulceration. At our institution, we have also documented GI dysmotility based on evaluation of gastric emptying in patients receiving bortezomib-based therapy. Promotility agents such as metoclopramide can be used in this situation, and attention to the avoidance of constipation is important. The International Myeloma Foundation (IMF) Nurse Leadership Board has also compiled comprehensive guidelines for the management of GI side effects in MM patients treated with novel therapies. ${ }^{65}$

\section{Herpes zoster reactivation}

Herpes zoster virus (HZV) reactivation within nerve cell bodies results in a characteristic painful, vesicular, dermatomal rash. It most often occurs in immunocompromised individuals, who receive prolonged corticosteroid therapy or other immunosuppressive drugs, and among recipients of solid-organ or stem cell transplantation. ${ }^{66} \mathrm{MM}$ is associated with deficiency in both humoral and cellular immunity, ${ }^{67}$ and as such predisposes affected individuals to infection. An increased incidence of HZV reactivation among MM patients has not been conclusively demonstrated but seems very likely based on available data. In the APEX trial, however, bortezomib therapy was associated with an increased incidence of herpes zoster reactivation as compared to high-dose dexamethasone ( $13 \%$ vs $5 \%, P=0.0002) .{ }^{34}$ The majority of these infections were grade $1 / 2$ in severity, and the incidence of higher grade HZV reactivation was similar in the two treatment arms ${ }^{68}$ While the biological mechanism 
underlying the apparent association between bortezomib and HZV reactivation has not been elucidated, it does not appear related to a direct effect of bortezomib on T-cells, ${ }^{69}$ mature lymphocytes, ${ }^{70}$ or viral replication. ${ }^{71}$ On the basis of evidence from the APEX study as well as our experience in treating patients with newly diagnosed disease, we routinely recommend antiviral prophylaxis using an agent such as acyclovir or valacyclovir.

\section{Future directions}

The successful use of bortezomib in MM has fueled interest in novel approaches to the application of this agent as well as in the development of new proteasome inhibitors. Combination therapy incorporating bortezomib together with emerging classes of drugs in MM are a key area of interest. As alluded to previously, the anti-MM mechanisms of bortezomib are synergistic with those of other drug classes (Figure 1), and combination therapy improves response and survival rates in comparison to single-agent therapy. In particular, the high level of activity observed to date with bortezomib-based combinations such as VTD, PAD, and RVD has provided the impetus for new approaches to therapy incorporating emerging drugs in MM. For example, the combinations of bortezomib and the histone deacetylase (HDAC) inhibitor vorinostat, ${ }^{72-74}$ bortezomib and the heat shock protein 90 (Hsp90) inhibitor tanespimycin, ${ }^{75}$ and bortezomib and the Akt pathway inhibitor perifosine ${ }^{76}$ have been evaluated in early phase clinical trials involving patients with relapsed and/or refractory MM. These regimens have shown promising anti-MM activity, even among patients who previously were refractory to bortezomib, and the respective toxicity profiles have been favorable. As additional classes of drugs gain a foothold in MM therapy, it will be necessary to determine the efficacy of certain regimens, but also the sequence and schedule by which agents are administered.

Historically, ASCT in MM has served as a means by which to increase the depth of response achieved through induction therapy. Indeed, ASCT following induction has been a standard of care for younger MM patients eligible for the procedure and is associated with a PFS advantage as compared to induction therapy alone in this group of patients. ${ }^{77}$ Whether induction regimens incorporating bortezomib and

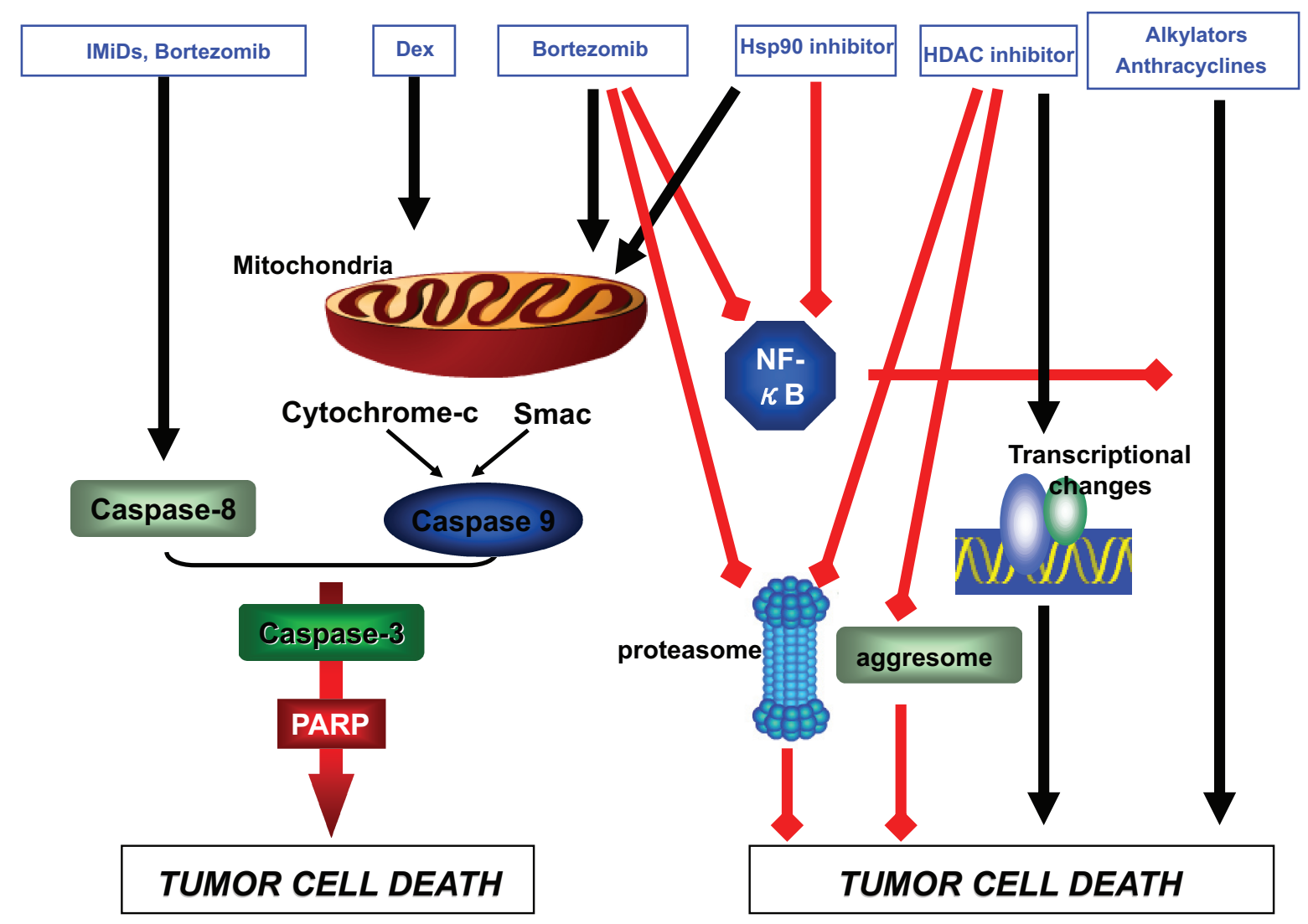

Figure I Synergistic anti-MM activity of bortezomib in combination with other agents.

Abbreviations: Hsp90 inhibitor, heat shock protein 90 inhibitor; HDAC inhibitor, histone deacetylase inhibitor; IMiD, immunomodulatory drugs; MM, multiple myeloma; PARP, poly(ADP-ribose)polymerase; Smac, second mitochondria-derived activator of caspase. 
other novel agents, which are associated with unprecedented response rates in this disease, will improve the relative benefit of ASCT in MM is unknown. That response rates among patients who receive bortezomib-based induction improve further in the aftermath of ASCT suggests this may be true, but the issue needs to be definitively assessed in a randomized comparison of novel therapy-based induction with or without ASCT consolidation.

The use of bortezomib maintenance therapy following ASCT is also an area of considerable interest. The practice in MM of post-ASCT maintenance therapy using thalidomide has been established on the basis of results from four randomized clinical trials that suggest the approach improves PFS. ${ }^{78-80}$ Moreover, results from three of these trials indicate that thalidomide maintenance is associated with an OS benefit. Caution must be exercised with this approach, however, as there is evidence thalidomide maintenance after ASCT may be associated with shorter survival times following relapse. ${ }^{78,81}$ Whether post-ASCT maintenance with bortezomib, through continued inhibition of the proteasome, confers significant clinical benefit is unknown. This question is being addressed as part of the previously referenced phase III trial led by the HOVON group, in which transplant-eligible individuals with newly diagnosed MM receive either VAD or PAD induction followed by ASCT and subsequently by maintenance thalidomide (in the VAD arm) or bortezomib administered every other week (in the PAD arm). ${ }^{45}$ As discussed previously, preliminary results indicate that bortezomib maintenance deepens the level of response to therapy. Updated analyses of this study are awaited with interest.

Finally, novel proteasome inhibitors are being developed with the aim of maintaining potent proteasome inhibition while modulating toxicity, specifically neurotoxicity, and altering bioavailability such that oral administration is possible. Two new proteasome inhibitors, carfilzomib (PR-171) and salinosporamide (NPI-0052), have to date undergone preclinical evaluation and are currently being assessed in early phase clinical trials involving MM patients with relapsed and/or refractory disease. ${ }^{82-84}$

\section{Conclusions}

As highlighted by this review, both bortezomib alone and in particular bortezomib-based combination therapy are important treatment options for individuals with newly diagnosed and relapsed MM. Bortezomib can be utilized safely in patients with renal dysfunction and has unique impact on bone disease frequently associated with MM. Moreover, the agent is effective in patients considered to have high-risk disease on the basis of chromosomal abnormalities and advanced ISS stage. The ability to safely and effectively partner bortezomib with other agents, both conventional and novel, is especially encouraging. In this context, treatment-associated toxicities associated with bortezomib are manageable with close monitoring and appropriate dose modifications as well as supportive care interventions. It is likely that with ongoing translational and clinical research efforts, patients with MM will, in the future, derive even greater benefit from bortezomib and the emerging second-generation proteasome inhibitors.

\section{Disclosures}

JP Laubach: Advisory Board Novartis Pharmaceuticals. CS Mitsiades: Consultant Millennium Pharmaceuticals, Novartis Pharmaceuticals, and Kosan Pharmaceuticals. RL Schlossman: Speakers Bureau Celgene Corporation and Millennium Pharmaceuticals. Nikhil Munshi: Speakers Bureau Celgene Corporation and Millennium Pharmaceuticals; Advisory Board Celgene Corporation, Millennium Pharmaceuticals, and Novartis Pharmaceuticals. IM Ghobrial: Speakers Bureau Celgene Corporation, Millennium Pharmaceuticals, and Novartis Pharmaceuticals; research support Celegene Corporation and Millennium Pharmaceuticals. KC Anderson: Advisory Board Celgene Corporation, Millennium Pharmaceuticals; consultant Celgene Corporation and Millennium Pharmaceuticals; research support Celgene Corporation and Millennium Pharmaceuticals. PG Richardson: Advisory Board Celgene Corporation, Millennium Pharmaceuticals; Speakers Bureau Celgene Corporation and Millennium Pharmaceuticals.

\section{References}

1. McKenna RW, Kyle RA, Kuehl WM, Grogan TM, Harris NL, Couplan RW. Plasma Cell Neoplasms. In: Swedlow SH, Campo E, Harris NL, et al. eds. WHO Classification of tumors of haematopoietic and lymphoid tissues. Lyon: International Agency for Research on Cancer; 2008.

2. Jemel A, Siegel R, Ward E, Hao Y, Xu J, Murray T, et al. Cancer Statistics. CA Cancer J Clin. 2008;58(2):71-96.

3. Barlogie B, Shaughnessy J, Epstein J, et al. Plasma Cell Myeloma. In: Lichtman MA, Beutler E, Kipps TJ, Seligsohn U, Kaushansky K, Prchal JT, editors. Williams Hematology. 7th ed. New York: McGraw-Hill; 2005. p.

4. Alexanian R, Barlogie B, Dixon D. High dose glucocorticoid treatment of resistant myeloma. Ann Intern Med. 1986;105(1):8-11.

5. Gertz MA, Garton JP, Greipp PR, Witzig TE, Kyle RA. A phase II study of high-dose methylprednisolone in refractory or relapsed multiple myeloma. Leukemia. 1995;9(12):2115-2118.

6. Oken MM, Harrington DP, Abramson N, Kyle RA, Knopse W, Glick JH. Comparison of melphalan and prednisone with vincristine, carmustine, melphalan, cyclophosphamide, and prednisone in the treatment of multiple myeloma - Results of Eastern Cooperative Oncology Group Study E2479. Cancer. 1997;79(8):1561-1567. 
7. Barlogie B, Smith L, Alexanian R. Effective treatment of advanced multiple myeloma refractory to alkylating agents. $N$ Engl J Med. 1984;310(21):1353-1356.

8. Anderson H, Scarffe JH, Ranson M, et al. VAD chemotherapy as remission induction for multiple myeloma. Br J Cancer. 1995;71(2): 326-330.

9. Gertz MA, Kalish LA, Kyle RA, Hahn RG, Tormey DC, Oken MM. Phase III study comparing vincristine, doxorubicin (Adriamycin), and dexamethasone (VAD) chemotherapy with VAD plus recombinant interferon alfa-2 in refractory or relapsed multiple myeloma. An Eastern Cooperative Oncology Group study. Am J Clin Oncol. 1995; 18(6):475-480.

10. Lokhorst HM, Meuwissen OJ, Bast EJ, Dekker AW. VAD chemotherapy for refractory multiple myeloma. $\mathrm{Br} J$ Haematol. 1989;71(1):25-30.

11. Phillips JK, Sherlaw-Johnson C, Pearce R, et al. A randomized study of MOD versus VAD in the treatment of relapsed and resistant multiple myeloma. Leuk Lymphoma. 1995;17(5-6):465-472.

12. Barlogie B, Hall R, Zander A, Dicke K, Alexanian R. High-dose melphalan with autologous bone marrow transplantation for multiple myeloma. Blood. 1986;67(5):1298-1301.

13. Barlogie B, Alexanian R, Dicke KA, et al. High-dose chemoradiotherapy and autologous bone marrow transplantation for resistant multiple myeloma. Blood. 1987;70(3):869-872.

14. Myung J, Kim KB, Crews CM. The ubiquitin-proteasome pathway and proteasome inhibitors. Med Res Rev. 2001;21(4):245-273.

15. Hideshima T, Richardson P, Chauhan D, et al. The proteasome inhibitor PS-341 inhibits growth, induces apoptosis, and overcomes drug resistance in human multiple myeloma cells. Cancer Res. 2001;61(7):3071-3076.

16. Hideshima T, Chauhan D, Richardson P, et al. NF-kappa B as a therapeutic target in multiple myeloma. J Biol Chem. 2002;277(19): 16639-16647.

17. Mitsiades N, Mitsiades CS, Richardson PG, et al. The proteasome inhibitor PS-341 potentiates sensitivity of multiple myeloma cells to conventional chemotherapeutic agents: therapeutic applications. Blood. 2003;101(6):2377-2380.

18. Hideshima T, Mitsiades C, Akiyama M, et al. Molecular mechanisms mediating antimyeloma activity of proteasome inhibitor PS-341. Blood. 2003;101(4):1530-1534.

19. Obeng EA, Carlson LM, Gutman DM, Harrington WJ Jr, Lee KP, Boise LH. Proteasome inhibitors induce a terminal unfolded protein response in multiple myeloma cells. Blood. 2006;107(12):4907-4916.

20. Dong H, Chen L, Chen X, et al. Dysregulation of unfolded protein response partially underlies proapoptotic activity of bortezomib in multiple myeloma cells. Leuk Lymphoma. 2009;50(6):974-984.

21. Hideshima T, Chauhan D, Hayashi T, et al. Proteasome Inhibitor PS-341 abrogates IL-6 triggered signaling cascades via caspasedependent downregulation of gp130 in multiple myeloma. Oncogene. 2003;22(52):8386-8393.

22. Kyle RA, Gertz MA, Witzig TE, et al. Review of 1027 patients with newly diagnosed multiple myeloma. Mayo Clin Proc. 2003; 78(1):21-33.

23. Lentzsch S, Ehrlich LA, Roodman GD. Pathophysiology of multiple myeloma bone disease. Hematol Oncol Clin North Am. 2007;21(6):1035-1049, viii.

24. Giuliani N, Rizzoli V, Roodman GD. Multiple myeloma bone disease: Pathophysiology of osteoblast inhibition. Blood. 2006;108(13): 3992-3996.

25. Terpos E, Heath DJ, Rahemtulla A, et al. Bortezomib reduces serum dickkopf-1 and receptor activator of nuclear factor-kappaB ligand concentrations and normalises indices of bone remodelling in patients with relapsed multiple myeloma. Br J Haematol. 2006;135(5): 688-692.

26. Terpos E, Sezer O, Croucher P, Dimopoulos MA. Myeloma bone disease and proteasome inhibition therapies. Blood. 2007;110(4): 1098-1104.
27. Zavrski I, Krebbel H, Wildemann B, et al. Proteasome inhibitors abrogate osteoclast differentiation and osteoclast function. Biochem Biophys Res Commun. 2005;333(1):200-205.

28. Mukherjee S, Raje N, Schoonmaker JA, et al. Pharmacologic targeting of a stem/progenitor population in vivo is associated with enhanced bone regeneration in mice. J Clin Invest. 2008;118(2):491-504.

29. Deleu S, Lemaire M, Arts J, et al. Bortezomib alone or in combination with the histone deacetylase inhibitor JNJ-26481585: effect on myeloma bone disease in the 5T2MM murine model of myeloma. Cancer Res. 2009;69(13):5307-5311.

30. Pennisi A, Li X, Ling W, Khan S, Zangari M, Yaccoby S. The proteasome inhibitor, bortezomib suppresses primary myeloma and stimulates bone formation in myelomatous and nonmyelomatous bones in vivo. $\mathrm{Am} \mathrm{J}$ Hematol. 2009;84(1):6-14.

31. Orlowski RZ, Stinchcombe TE, Mitchell BS, et al. Phase I trial of the proteasome inhibitor PS-341 in patients with refractory hematologic malignancies. J Clin Oncol. 2002;20(22):4420-4427.

32. Richardson PG, Barlogie B, Berenson J, et al. A phase 2 study of bortezomib in relapsed, refractory myeloma. $N$ Engl J Med. 2003; 348(26):2609-2617.

33. Jagannath S, Barlogie B, Berenson J, et al. A phase 2 study of two doses of bortezomib in relapsed or refractory myeloma. Br J Haematol. 2004;127(2):165-172.

34. Richardson PG, Sonneveld P, Schuster MW, et al. Bortezomib or high-dose dexamethasone for relapsed multiple myeloma. $N$ Engl $J$ Med. 2005;352(24):2487-2498.

35. Richardson PG, Sonneveld P, Schuster M, et al. Extended follow-up of a phase 3 trial in relapsed multiple myeloma: final time-to-event results of the APEX trial. Blood. 2007;110(10):3557-3560.

36. Ma MH, Yang HH, Parker K, et al. The proteasome inhibitor PS-341 markedly enhances sensitivity of multiple myeloma tumor cells to chemotherapeutic agents. Clin Cancer Res. 2003;9(3):1136-1144.

37. Orlowski RZ, Nagler A, Sonneveld P, et al. Randomized phase III study of pegylated liposomal doxorubicin plus bortezomib compared with bortezomib alone in relapsed or refractory multiple myeloma: combination therapy improves time to progression. J Clin Oncol. 2007;25(25):3892-3901.

38. Palumbo A, Gay F, Bringhen S, et al. Bortezomib, doxorubicin and dexamethasone in advanced multiple myeloma. Ann Oncol. 2008;19(6):1160-1165.

39. Pineda-Roman M, Zangari M, van Rhee F, et al. VTD combination therapy with bortezomib-thalidomide-dexamethasone is highly effective in advanced and refractory multiple myeloma. Leukemia. 2008;22(7):1419-1427.

40. Mitsiades N, Mitsiades CS, Poulaki V, et al. Molecular sequelae of proteasome inhibition in human multiple myeloma cells. Proc Natl Acad Sci US A. 2002;99(22):14374-14379.

41. Richardson P, Jagannath S, Jakubowiak A, et al. Lenalidomide, bortezomib, and dexamethasone in patients with relapsed or relapsed/ refractory multiple myeloma (MM): encouraging response rates and tolerabilitywith correlation of outcome and adverse cytogenetics in a phase II study. American Society of Hematology Annual Meeting; 2008. Abstract 1742.

42. San Miguel JF, Schlag R, Khuageva NK, et al. Bortezomib plus melphalan and prednisone for initial treatment of multiple myeloma. N Engl J Med. 2008;359(9):906-917.

43. Harousseau JL, Mathiot C, Attal M, et al. Bortezomib/dexamethasone versus VAD as induction prior to autologous stem cell transplantation (ASCT) in previously untreated multiple myeloma (MM): updated data from IFM2005/01 trial. American Society of Clinical Oncology Annual Meeting; 2008. Abstract 8505.

44. Cavo M, Tacchetti P, Patriarca F, et al. Superior complete response rate and progression-free survival after autologous transplantation with up-front velcade-thalidomide-dexamethasone compared with thalidomide-dexamethasone in newly diagnosed multiple myeloma. American Society of Hematology, Annual Meeting; 2008. Abstract 158 . 
45. Sonneveld P, Van der Holt B, Schmidt-Wolf I, et al. First analysis of HOVON-65/GMMG-HD4 randomized phase III trial comparing bortezomib, adriamycine, dexamethasone (PAD) vs VAD as induction treartment prior to high dose melphalan (HDM) in patients with newly diagnosed multiple myeloma (MM). American Society of Hematology Annual Meeting; 2008. Abstract 653.

46. Richardson P, Lonial S, Jakubowiak A, et al. Lenalidomide, bortezomib, and dexamethasone in patients with newly diagnosed multiple myeloma: encouraging efficacy in high risk groups with updated results of a phase I/II study. American Society of Hematology, Annual Meeting; 2008. Abstract 92

47. Richardson P, Lonial S, Jakubowiak A, et al. Lenalidomide, bortezomib, and dexamethasone has notable activity in high-risk first-line myeloma. 2009 International Myeloma Workshop; 2009. Abstract 224.

48. National Comprehensive Cancer Network. NCCN clinical practice guidelines in oncology. Multiple Myeloma. v.2.2009. URL: www. nccn.org.

49. Richardson PG, Barlogie B, Berenson J, et al. Clinical factors predictive of outcome with bortezomib in patients with relapsed, refractory multiple myeloma. Blood. 2005;106(9):2977-2981.

50. Jagannath S, Richardson PG, Sonneveld P, et al. Bortezomib appears to overcome the poor prognosis conferred by chromosome 13 deletion in phase 2 and 3 trials. Leukemia. 2007;21(1):151-157.

51. Chang H, Trieu Y, Qi X, Xu W, Stewart KA, Reece D. Bortezomib therapy response is independent of cytogenetic abnormalities in relapsed/ refractory multiple myeloma. Leuk Res. 2007;31(6):779-782.

52. Sagaster V, Ludwig H, Kaufmann H, et al. Bortezomib in relapsed multiple myeloma: response rates and duration of response are independent of a chromosome 13q-deletion. Leukemia. 2007;21(1): 164-168.

53. Blade J, Fernandez-Llama P, Bosch F, et al. Renal failure in multiple myeloma: presenting features and predictors of outcome in 94 patients from a single institution. Arch Intern Med. 1998;158(17):1889-1893.

54. Chanan-Khan AA, Kaufman JL, Mehta J, et al. Activity and safety of bortezomib in multiple myeloma patients with advanced renal failure: a multicenter retrospective study. Blood. 2007;109(6):2604-2606.

55. Wechalekar AD, Lachmann HJ, Offer M, Hawkins PN, Gillmore JD. Efficacy of bortezomib in systemic AL amyloidosis with relapsed/ refractory clonal disease. Haematologica. 2008;93(2):295-298.

56. Argyriou AA, Iconomou G, Kalofonos HP. Bortezomib-induced peripheral neuropathy in multiple myeloma: a comprehensive review of the literature. Blood. 2008;112(5):1593-1599.

57. Cavaletti G, Nobile-Orazio E. Bortezomib-induced peripheral neurotoxicity: still far from a painless gain. Haematologica. 2007;92(10):1308-1310.

58. Richardson PG, Briemberg H, Jagannath S, et al. Frequency, characteristics, and reversibility of peripheral neuropathy during treatment of advanced multiple myeloma with bortezomib. J Clin Oncol. 2006;24(19):3113-3120.

59. Badros A, Goloubeva O, Dalal JS, et al. Neurotoxicity of bortezomib therapy in multiple myeloma: a single-center experience and review of the literature. Cancer. 2007;110(5):1042-1049.

60. Packer L, Kraemer K, Rimbach G. Molecular aspects of lipoic acid in the prevention of diabetes complications. Nutrition. 2001;17(10): $888-895$.

61. De Grandis D. Acetyl-L-carnitine for the treatment of chemotherapyinduced peripheral neuropathy: a short review. CNS Drugs 2007; 21 Suppl 1:39-43; discussion 5-6.

62. Colvin LA, Johnson PR, Mitchell R, Fleetwood-Walker SM, Fallon M. From bench to bedside: a case of rapid reversal of bortezomib-induced neuropathic pain by the TRPM8 activator, menthol. J Clin Oncol. 2008;26(27):4519-4520.

63. Cleary JF. The pharmacologic management of cancer pain. $J$ Palliat Med. 2007;10(6):1369-1394.

64. Lonial S, Waller EK, Richardson PG, et al. Risk factors and kinetics of thrombocytopenia associated with bortezomib for relapsed, refractory multiple myeloma. Blood. 2005;106:3777-3784.
65. Smith LC, Bertolotti P, Curran K, Jenkins B. Gastrointestinal side effects associated with novel therapies in patients with multiple myeloma: consensus statement of the IMF Nurse Leadership Board. Clin J Oncol Nurs. 2008;12 Suppl 3:37-52.

66. Arvin AM. Varicella-Zoster virus: pathogenesis, immunity, and clinical management in hematopoietic cell transplant recipients. Biol Blood Marrow Transplant. 2000;6(3):219-230.

67. Schutt P, Brandhorst D, Stellberg W, Poser M, Ebeling P, Muller S, et al. Immune parameters in multiple myeloma patients: influence of treatment and correlation with opportunistic infections. Leuk Lymphoma. 2006;47(8):1570-1582.

68. Chanan-Khan A, Sonneveld P, Schuster MW, Stadtmauer EA, Facon T, Harousseau JL, et al. Analysis of herpes zoster events among bortezomib-treated patients in the phase III APEX study. J Clin Oncol. 2008;26(29):4784-4790.

69. Blanco B, Perez-Simon JA, Sanchez-Abarca LI, et al. Bortezomib induces selective depletion of alloreactive $\mathrm{T}$ lymphocytes and decreases the production of Th1 cytokines. Blood. 2006;107(9): 3575-3583.

70. Maseda D, Meister S, Neubert K, Herrmann M, Voll RE. Proteasome inhibition drastically but reversibly impairs murine lymphocyte development. Cell Death Differ. 2008;15(3):600-612.

71. Neznanov N, Dragunsky EM, Chumakov KM, et al. Different effect of proteasome inhibition on vesicular stomatitis virus and poliovirus replication. PLoS ONE 2008;3(4):e1887.

72. Richardson P, Mitsiades C, Colson K, et al. Phase I trial of oral vorinostat (suberoylanilide hydroxamic acid, SAHA) in patients with advanced multiple myeloma. Leuk Lymphoma. 2008;49(3): 502-507.

73. Weber D, Badros AZ, Jagannath S, et al. Vorinostat plus bortezomib for the treatment of relapsed/refractory multiple Mmyeloma: early clinical experience. Blood. 2008;102:322.

74. Badros A, Philip S, Niesvizky R, et al. Phase I trial of suberoylanilide hydroxamic acid (SAHA) + bortezomib (bort) in relapsed multiple myeloma (MM) patients. American Society of Hematology Annual Meeting; 2007. Abstract 1168.

75. Richardson PG, Chanan-Khan A, Lonial S, et al. Tanespimycin $(\mathrm{T})$ + bortezomib (bz) in multiple myeloma (MM): confirmation of the recommended dose using a novel formulation. American Society of Hematology Annual Meeting. Blood. 2007;110: Abstract 1165.

76. Richardson P, Wolf J, Jakubowiak A, et al. Phase I/II results of a multicenter trial of perifosine (KRX-0401) + bortezomib in patients with relapsed or relapsed / refractory multiple myeloma who were previously relapsed from or refractory to bortezomib. Blood. 2008;102:321.

77. Koreth J, Cutler CS, Djulbegovic B, et al. High-dose therapy with single autologous transplantation versus chemotherapy for newly diagnosed multiple myeloma: A systematic review and meta-analysis of randomized controlled trials. Biol Blood Marrow Transplant. 2007; 13(2):183-196.

78. Barlogie B, Tricot G, Anaissie E, et al. Thalidomide and hematopoieticcell transplantation for multiple myeloma. $N$ Engl J Med. 2006;354(10): 1021-1030.

79. Attal M, Harousseau JL, Leyvraz S, et al. Maintenance therapy with thalidomide improves survival in patients with multiple myeloma. Blood. 2006;108(10):3289-3294.

80. Spencer A, Prince HM, Roberts AW, et al. Consolidation Therapy With Low-Dose Thalidomide and Prednisolone Prolongs the Survival of Multiple Myeloma Patients Undergoing a Single Autologous Stem-Cell Transplantation Procedure. J Clin Oncol. 2009;27(11): 1788-1793.

81. Morgan GJ, Jackson GH, Davies FE, et al. Maintenance thalidomide may improve progression free but not overall survival; results from the myeloma IX maintenance randomisation. American Society of Hematology Annual Meeting. Blood. 2008;112: Abstract 656.

82. Richardson P, Hofmeister CC, Zimmerman TM, et al. Phase 1 clinical trial of NPI-0052, a novel proteasome inhibitor in patients with multiple myeloma. Blood. 2008;112: Abstract 2770. 
83. Vij R, Wang M, Orlowski R, et al. Initial results of PX-171-004, an openlabel, single-arm, phase II study of carfilzomib (CFZ) in patients with relapsed myeloma (MM). American Society of Hematology, Annual Meeting. Blood. 2008;112: Abstract 865.
84. Jagannath S, Vij R, Stewart AK, et al. Initial results of PX-171-003, an open-label, single-arm, phase II study of carfilzomib (CFZ) in patients with relapsed and refractory multiple myeloma (MM). Blood. 2008;112: Abstract 864 .

\section{Publish your work in this journal}

Cancer Management and Research is an international, peer-reviewed open access journal focusing on cancer research and the optimal use of preventative and integrated treatment interventions to achieve improved outcomes, enhanced survival and quality of life for the cancer patient The journal welcomes original research, clinical \& epidemiological studies, reviews \& evaluations, guidelines, expert opinion \& commentary, case reports \& extended reports. The manuscript management system is completely online and includes a very quick and fair peerreview system, which is all easy to use. Visit http://www.dovepress.com/ testimonials.php to read real quotes from published authors.

Submit your manuscript here: http://www.dovepress.com/cancer-management-and-research-journal 